\title{
CONFIDENCE INTERVALS CONSTRUCTED BY MODEL AVERAGING AND BOOTSTRAP SMOOTHING
}

\author{
CHRISTEEN WIJETHUNGA (D)
}

(Received 27 May 2020; first published online 11 September 2020)

2020 Mathematics subject classification: primary 62F25; secondary 62C99, 62F40, 62J07.

Keywords and phrases: confidence interval, model averaged estimator, bootstrap smoothed estimator, coverage probability, scaled expected length.

We assess the performance of confidence intervals constructed by model averaging and bootstrap smoothing, using a simple testbed situation of two nested linear regression models with either known error variance or unknown error variance. The assessment is carried out by deriving computationally convenient exact expressions for the coverage probability and scaled expected length where the scaling is with respect to the usual confidence interval, with the same minimum coverage, based on the full model.

We assess the properties of the confidence interval centred on the model averaged estimator proposed by Buckland et al. [1] with half-width proportional to the standard error of the estimate put forward in [1, formula (9)]. We consider both the finite sample case and the asymptotic case. Our results show that this model averaged confidence interval cannot be generally recommended, although it performs well when the error degrees of freedom is 1 .

We also assess the properties of the confidence intervals centred on the bootstrap smoothed estimator with half-width proportional to the estimate of (a) the standard deviation of this estimator and (b) the delta method approximation, derived by Efron [2], to the standard deviation of this estimator. We show that, except for the case of unknown error variance and small error degrees of freedom, the confidence interval centred on the bootstrap smoothed estimator does not have scaled expected length that is substantially less than 1, when the simpler model is correct. For this reason, we sought a formula for the data-based width that leads to improved performance of the confidence interval centred on the bootstrap smoothed estimator. Since this search failed, it raises the following question. Is there any formula for the data-based width of such a confidence interval so that it performs well? To answer this question, we compute the performance bound due to Kabaila and Kong [3].

Thesis submitted to La Trobe University in September 2019; degree approved on 11 December 2019; supervisor Paul Kabaila, co-supervisor David Farchione.

(C) 2020 Australian Mathematical Publishing Association Inc. 


\section{References}

[1] S. T. Buckland, K. P. Burnham and N. H. Augustin, 'Model selection: an integral part of inference', Biometrics 53 (1997), 603-618.

[2] B. Efron, 'Estimation and accuracy after model selection', J. Am. Stat. Assoc. 109 (2014), 991-1007.

[3] P. Kabaila and Y. Kong, 'Lower bounds on integrated risk, subject to inequality constraints', Aust. N. Z. J. Stat. 58 (2016), 293-315.

CHRISTEEN WIJETHUNGA, Department of Mathematics and Statistics, La Trobe University, Bundoora, Victoria 3086, Australia

e-mail: christeen.wijethunga@gmail.com 